\title{
Investigation of heat and mass transfer between the two phases of an evaporating droplet stream using laser-induced fluorescence techniques: Comparison with modeling
}

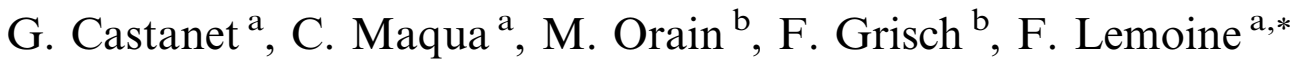 \\ ${ }^{a}$ LEMTA-CNRS UMR 7563, 2, Avenue de la forêt de Haye, BP 160, 54504 Vandoeuvre-lès-Nancy, France \\ ${ }^{\mathrm{b}}$ Office National d'Etudes et de Recherches Aérospatiales, Fort de Palaiseau, 91761 Palaiseau Cedex, France
}

\begin{abstract}
Heat and mass exchanges between the two phases of a spray is a key point for the understanding of physical phenomena occurring during spray evaporation in a combustion chamber. Development and validation of physical models and computational tools dealing with spray evaporation requires experimental databases on both liquid and gas phases. This paper reports an experimental study of evaporating acetone droplets streaming linearly at moderate ambient temperatures up to $75^{\circ} \mathrm{C}$. Two-color laser-induced fluorescence is used to characterize the temporal evolution of droplet mean temperature. Simultaneously, fuel vapor distribution in the gas phase surrounding the droplet stream is investigated using acetone planar laser-induced fluorescence.

Temperature measurements are compared to simplified heat and mass transfer model taking into account variable physical properties, droplet-to-droplet interactions and internal fluid circulation within the droplets. The droplet surface temperature, calculated with the model, is used to initiate the numerical simulation of fuel vapor diffusion and transport in the gas phase, assuming thermodynamic equilibrium at the droplet surface. Influence of droplet diameter and droplet spacing on the fuel vapor concentration field is investigated and numerical results are compared with experiments.
\end{abstract}

Keywords: Laser-induced fluorescence; Droplet; Acetone; Evaporation; Temperature; Fuel mass fraction

\section{Introduction}

Most industrial combustors operate with liquid fuel sprays (e.g. aircraft engines, gas turbines) in order to improve combustion efficiency by increasing heat and mass transfer surface between fuel and oxidizer. However, spray vaporization and combustion are very complex topics which require the understanding of physical processes such as spray atomization, transport by turbulence, chemistry and also interactions of all these quantities. For instance, depending on parameters such as droplet diameter and spacing, the fuel spatial distribution around droplets may change and various combustion regimes may occur such

\footnotetext{
* Corresponding author. Tel.: +33 3839957 32; fax: +33 383999944. E-mail address: fabrice.lemoine@ensem.inpl-nancy.fr (F. Lemoine).
}

as group combustion or individual droplet burning [1,2]. In addition, spatial and temporal non-uniformities of fuel distribution have been identified as key factors leading to increased pollutant emissions or combustion oscillations $[3,4]$. Nonetheless, in an industrial-type combustor, all the parameters mentioned above are generally coupled to each other, which makes it difficult to determine their separate influence. As a result, experiments are often performed on idealized sprays in order to minimize the coupling between the different effects and to provide parametric results. In particular, many studies on droplet evaporation or combustion have been performed using linear monodisperse droplet streams [5-8]. This simple geometry is particularly attractive because it generates a temporally and spatially repeatable stream of droplets with well-controlled injection frequency, droplet size and velocity and 


\begin{tabular}{|c|c|c|c|}
\hline \multicolumn{4}{|c|}{ Nomenclature } \\
\hline$a$ & thermal diffusivity & $t$ & time \\
\hline$A$ & Einstein coefficient of spontaneous emission & $U_{\mathrm{S}}$ & maximum surface velocity \\
\hline$B$ & Einstein coefficient of absorption & $V$ & probe volume \\
\hline$B_{\mathrm{M}}$ & mass transfer Spalding number, $B_{\mathrm{M}}=\frac{Y_{\mathrm{S}}-Y_{\infty}}{1-Y_{\mathrm{S}}}$ & $\vec{V}$ & gas phase velocity vector \\
\hline C & reduced droplet spacing or distance parameter & $V_{\mathrm{d}}$ & droplet velocity \\
\hline$C_{p}$ & specific heat & $Y_{\mathrm{K}}$ & fuel vapor mass fraction \\
\hline$D$ & droplet diameter & & \\
\hline$D_{\mathrm{g}}$ & molecular diffusivity of acetone vapor & \multicolumn{2}{|c|}{ Greek symbols } \\
\hline E & laser energy & $\alpha$ & optical constant \\
\hline$f_{i}$ & population fraction of the pumped states & $\delta$ & fuel vapor boundary layer thickness \\
\hline$I$ & fluorescence intensity & $\Phi_{\mathrm{C}}$ & convective heat flux \\
\hline Le & Lewis number & $\Phi_{\text {vap }}$ & vaporization heat flux \\
\hline$L_{\mathrm{v}}$ & liquid fuel latent heat of vaporization & $\chi$ & fuel vapor molar fraction \\
\hline$m$ & droplet mass & $\lambda$ & thermal conductivity \\
\hline$\dot{m}$ & fuel vapor flowrate & $\mu$ & dynamic viscosity \\
\hline$N_{\mathrm{abs}}$ & number density of absorbing species & $\rho$ & specific density \\
\hline $\mathrm{Nu}$ & space averaged Nusselt number & & \\
\hline $\operatorname{Pr}$ & Prandtl number & \multicolumn{2}{|c|}{ Subscripts } \\
\hline$Q_{\mathrm{L}}$ & heat flux penetrating into the droplet & $*$ & normalized quantities \\
\hline$P$ & pressure & $\infty$ & conditions far from the droplets \\
\hline$Q$ & collisional quenching rate & $\mathrm{d}$ & droplet \\
\hline $\operatorname{Re}$ & droplet Reynolds number & $\mathrm{g}$ & gas phase \\
\hline$P e$ & Peclet number & iso & isolated droplet \\
\hline$r$ & radial coordinate & i & injection \\
\hline$R$ & droplet radius & $\mathrm{K}$ & fuel vapor phase \\
\hline$S$ & droplet surface & 1 & fuel liquid phase \\
\hline$S h$ & space averaged Sherwood number & ref & reference state \\
\hline$T$ & temperature & $\mathrm{S}$ & conditions at the droplet surface \\
\hline$T_{\mathrm{m}}$ & droplet mean temperature & & \\
\hline
\end{tabular}

droplet spacing, which allows determining the individual influence of each of these parameters on droplet vaporization. Therefore, experiments performed with this geometry can provide a database for various aerothermal conditions, which is very useful for the validation of computational fluid dynamics codes on test cases where all the flow parameters are controlled. In addition, physical models can be derived from such experiments and can be subsequently implemented into simulation codes in order to calculate more complex situations. For example, computation of the spatial distribution of fuel vapor concentration in the gas phase requires the knowledge of the vapor mass flowrate at the droplet surface, which is directly related to the liquid phase temperature. Therefore, it is necessary to collect experimental data simultaneously on both the liquid and gas phases in order to fully characterize the spray behavior for the validation of such computations. However, experimental studies found in the literature were focused either on the liquid fuel phase by measuring droplet size, velocity, temperature and mass flux or on fuel vapor surrounding the droplets, but very few provide combined measurements on both the liquid and gas phases. Measurement of physical parameters in a spray requires the use of non-intrusive techniques. One of the most usual techniques is Laser-Induced Fluorescence (LIF), which is particularly interesting because it allows discriminating the liquid and vapor phases of the fuel. In addition, LIF signals are intense, which leads to better detectivity and also improved signal-to-noise ratio. Spectroscopic properties of acetone are well known and, therefore, it has been widely used as a tracer in planar laser-induced fluorescence (PLIF) measurements of gas phase concentration and temperature $[9,10]$. In particular, spatial distribution of acetone vapor around a monodisperse stream of acetone droplets can be measured using acetone PLIF [11], while droplet mean temperature can be obtained with two-color laserinduced fluorescence [12].

The remaining paper comprises four sections. The first part describes the laser-induced fluorescence techniques and the optical set-up used in the experiments. The second and the third parts present the modeling for the liquid and gas phase with a special emphasis on boundary conditions. In the fourth part, experimental data obtained using combined measurements of fuel vapor concentration around a monodisperse stream of acetone droplets and droplet temperature are presented. The data are compared with 
calculations of the liquid phase temperature based on evaporation models of interacting droplets. The determined droplet surface temperature is then used as a boundary condition for numerical simulations of the gas phase taking into account vapor diffusion and transport. The computed vapor distribution is then compared to experimental results obtained by PLIF. Influence of droplet diameter and droplet spacing on the fuel vapor boundary layer is particularly examined for ambient temperature limited to $75^{\circ} \mathrm{C}$. Finally, the paper ends with a summary of the main findings.

\section{Experimental set-up and measurement techniques}

\subsection{Monodisperse droplet stream}

A linear monodisperse droplet stream is generated by Rayleigh disintegration of a liquid jet, with the use of a mechanical vibration obtained by a piezoceramic orifice [13]. The fuel can be pre-heated within the injector by means of an external heated water circulation. Fuel temperature is measured at the injection point with the use of a K type thermocouple. Additionally, the droplet stream can be injected in a large enclosure equipped with adequate optical access, where temperature can be maintained up to $75{ }^{\circ} \mathrm{C}$.

\subsection{Measurement techniques}

\subsubsection{Two-colors laser-induced fluorescence applied to droplet temperature measurements}

The main outlines of the two-color laser-induced fluorescence technique are presented in this section, and the extension of the method to acetone droplet temperature measurements is emphasized. Further technical details can be found in a previous paper [12]. Acetone fuel is previously seeded with a low concentration (a few $\mathrm{mg} / \mathrm{l}$ ) of rhodamine B. The fluorescence of rhodamine B, which can be easily induced by the green line of an argon ion laser $(\lambda=514.5 \mathrm{~nm})$, is broadband, and its temperature sensitivity is strongly dependent on the wavelength [12]. The fluorescence intensity, as a function of the different physical and optical parameters, is given by [14]:

$I_{\mathrm{f}}(\lambda)=K_{\text {opt }}(\lambda) K_{\text {spec }}(\lambda) V_{\mathrm{c}} I_{0} C \mathrm{e}^{\beta(\lambda) / T}$

where $K_{\text {opt }}(\lambda)$ is an optical constant, $K_{\text {spec }}(\lambda)$ is a constant depending solely on the spectroscopic properties of the fluorescent tracer in its environment (i.e. the fuel), $I_{0}$ the laser intensity, $C$ the molecular tracer concentration, $T$ the absolute temperature, $V_{\mathrm{c}}$ is the fluorescence photons collection volume. The product $C V_{\mathrm{c}}$ of the collection volume by the tracer molecular concentration is related to the number of fluorescence photons emitted by the rhodamine B molecules excited by the laser radiation and reaching the photodetector surface. This parameter is strongly dependent on the droplet size and on its position within the laser probe volume. The factor $\beta(\lambda)$ characterizes the temperature dependence of fluorescence intensity at the wavelength $\lambda$.

In order to collect a large number of photons, the fluorescence signal is detected over a spectral band $\left[\lambda_{i 1} ; \lambda_{i 2}\right], i$ denoting the spectral band. The resulting signal can be expressed using second-order polynomials as [7]:

$$
\int_{\lambda i 1}^{\lambda i 1} K_{\text {opt }}(\lambda) K_{\text {spec }}(\lambda) V_{\mathrm{c}} I_{0} C \mathrm{e}^{\beta(\lambda) / T} \mathrm{~d} \lambda=K_{\text {opt } i} K_{\text {speci }} V_{\mathrm{c}} I_{0} C \mathrm{e}^{\frac{a_{i}}{T^{2}} \frac{b_{i}}{T}}
$$

where $K_{\mathrm{opt} i}$ and $K_{\text {spec } i}$ are the optical and spectroscopic constants related to the spectral band $i$. The parameters $a_{i}$ and $b_{i}$ are the temperature sensitivity coefficients for the spectral band $i$. Measuring the temperature of a moving and evaporating droplet requires to eliminate the influence of the parameters $C V_{\mathrm{c}}$ and $I_{0}$. For example, in pure vaporization conditions, the dye concentration is likely to vary and the collection volume is constantly changing when the droplet crosses the probe volume. Furthermore, the intensity distribution of the laser inside the droplet depends on the position of the droplet within the probe volume due to the lensing effect of the droplet spherical interface. In order to overcome these problems, fluorescence intensity is detected over two spectral bands for which temperature sensitivity is highly different. Selection of these spectral bands is optimized with a preliminary study of the influence of temperature on the fluorescence spectrum of rhodamine B dissolved in acetone, which allows $\beta(\lambda)$ to be determined, as presented in Fig. 1. A first band located in the vicinity of $535 \mathrm{~nm}$ and a second one centered at $580 \mathrm{~nm}$ seems to be a good trade-off between signal intensity and temperature sensitivity. The ratio between both fluorescence intensities collected on both optimal spectral bands is given by:

$R_{\mathrm{f}}=\frac{I_{\mathrm{f} 1}}{I_{\mathrm{f} 2}}=\frac{K_{\mathrm{opt} 1}}{K_{\mathrm{opt} 2}} \frac{K_{\mathrm{spec} 1}}{K_{\mathrm{spec} 2}} \mathrm{e}^{\frac{a_{1}-a_{2}}{T^{2}}+\frac{b_{1}-b_{2}}{T}}$

This ratio is totally independent of the dimensions of the intersection between the droplet, the laser probe volume and the photon collection volume. The influence of the local laser intensity and tracer concentration is also totally eliminated. The set of coefficients $\left(a_{1}-a_{2}\right)$ and $\left(b_{1}-b_{2}\right)$ is preliminarily determined in a temperature-controlled cell. The use of a single reference point where temperature is known allows eliminating the optical and spectroscopic constants $K_{\text {opt }}$ and $K_{\text {spec. }}$.

The measurement volume is formed by means of a laser Doppler anemometer, which also allows the droplet velocity to be measured (Fig. 2). Droplet mean temperature can be obtained using a measurement volume diameter comparable to the droplet diameter itself and by averaging the fluorescence signal over the overall transit time of the droplet in the measurement volume [12]. The fluorescence signal is transmitted by an optical fiber to a set of beamsplitters and optical filters, which permits the fluorescence signal to be divided into the two aforementioned spectral bands. Mie scattering is eliminated using a high-pass filter. 


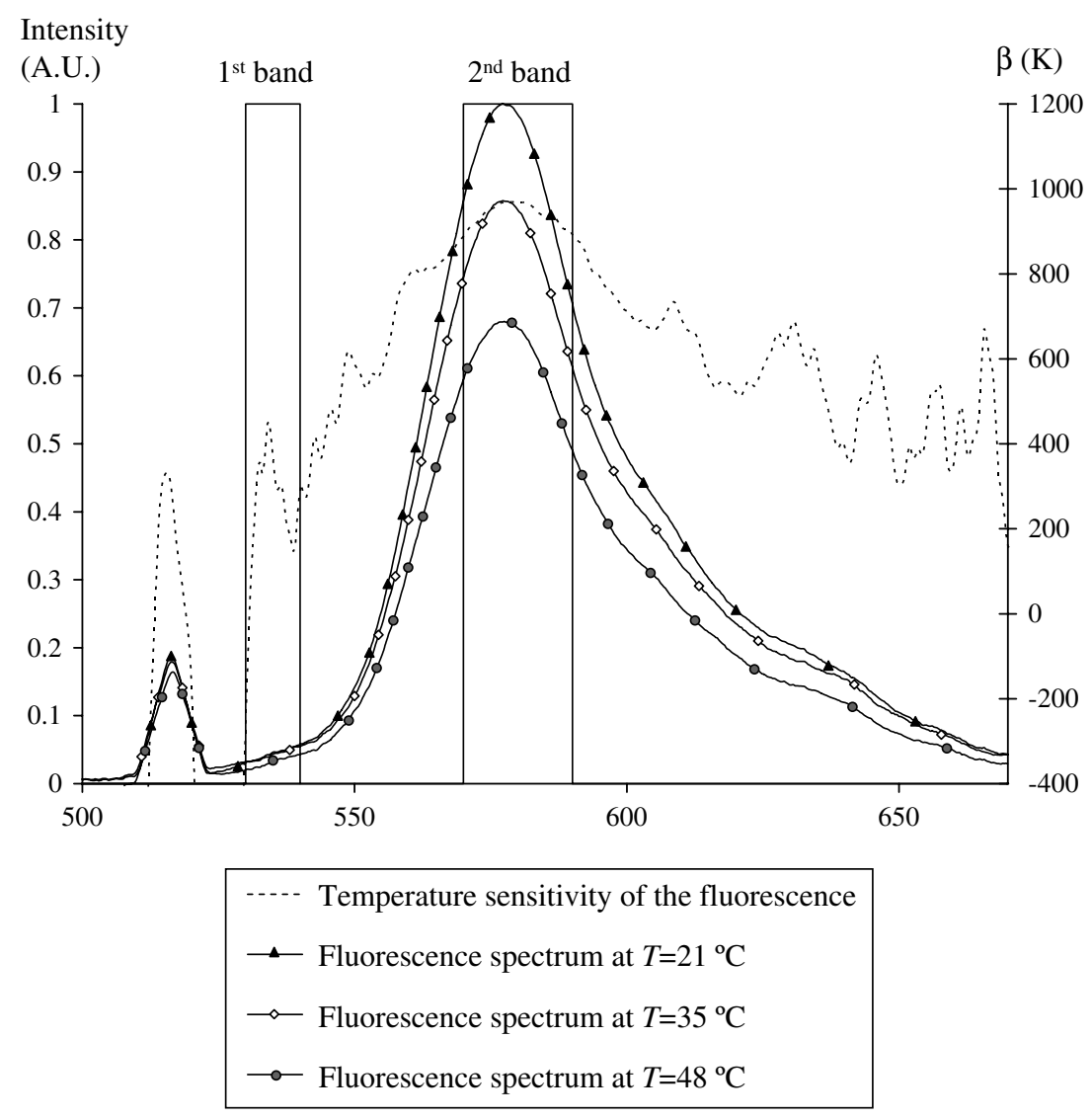

Fig. 1. Dependence of the temperature sensitivity $\beta(\lambda)$ as a function of fluorescence wavelength.

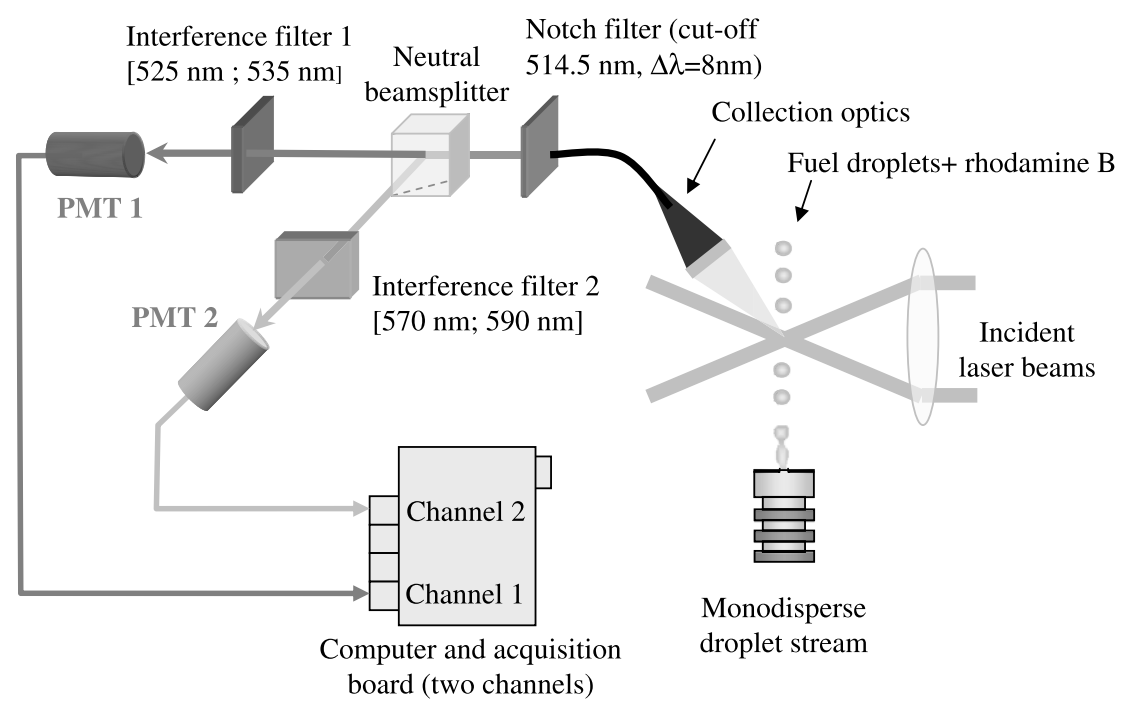

Fig. 2. Two-colors laser-induced fluorescence optical set-up.

The fluorescence signal is detected over the two spectral bands by means of two photomultiplier tubes equipped with two rapid pre-amplifiers (Fig. 2).

\subsubsection{Acetone vapor concentration measurements using}

Planar Laser-Induced Fluorescence (PLIF)

In the present experiment, acetone acts as the evaporating fuel as well as a fluorescent tracer for the gas phase. The main advantages of using acetone as a fluorescent tracer are summarized in [11].

PLIF is based on the absorption of the incident light by molecules which are excited to a higher energy level. Some of the excited molecules return to equilibrium by emitting fluorescence. The intensity of the fluorescence signal is proportional to the molar fraction $C$ of the absorbing fluorescent molecules and an efficiency coefficient depending on 
the temperature $T$ and the excitation wavelength $\lambda$. The flow is illuminated by a laser sheet which wavelength is tuned to excite a particular transition of a molecular species. The total light emission per unit sheet area, for unsaturated excitation is given by [15]:

$I=\alpha E V N_{\mathrm{abs}} f_{i}(T) B[A /(A+Q)]$

where $\alpha$ is a specific constant of the experimental set-up, $E$ is the laser energy per pulse per unit area per unit frequency, $V$ is the measurement volume, $N_{\text {abs }}$ is the number density of the absorbing species, and $f_{i}(T)$ represents the population fraction of the pumped states. $A$ and $B$ are the Einstein coefficients related to the spontaneous emission and absorption processes respectively. The parameter $Q$, known as the collisional quenching rate constant, represents the energy loss by both intermolecular redistribution and internal conversion. When a molar fraction is to be measured, a single-wavelength excitation tuned on a quantum state, which minimises the overall temperature dependence of the fluorescence signal in the temperature range of the experiment, can be used. Consequently, if pressure is constant, the fluorescence signal is proportional to the number density of fluorescent species. The proportionality factor can then be evaluated using suitable calibration techniques. It depends on experimental parameters such as probe volume, solid angle, spectral and spatial efficiencies of filters and cameras, power density of the laser, spectroscopic constants such as transition probability, absorption lineshape and fluorescence quantum yield which are usually known. The UV beam at $266 \mathrm{~nm}$, is formed into a thin sheet by combining cylindrical and spherical lenses (Fig. 3). Fluorescence from part of the laser sheet is collected by an imaging lens at right angles and recorded with a 16-bit intensified CCD camera equipped with a $512 \times 512$ array, connected to a PC, where the images are stored. The exposure time of the ICCD camera is $1 \mu \mathrm{s}$ and acquisition frequency is $2 \mathrm{~Hz}$. The area of the laser sheet imaged by the ICCD camera is equal to $8.5 \times 8.5 \mathrm{~mm}^{2}$ leading to spatial resolution of $16 \mu \mathrm{m}$ per pixel. Thickness of the laser sheet is of the order $130 \mu \mathrm{m}$. Spectral filters are placed in front

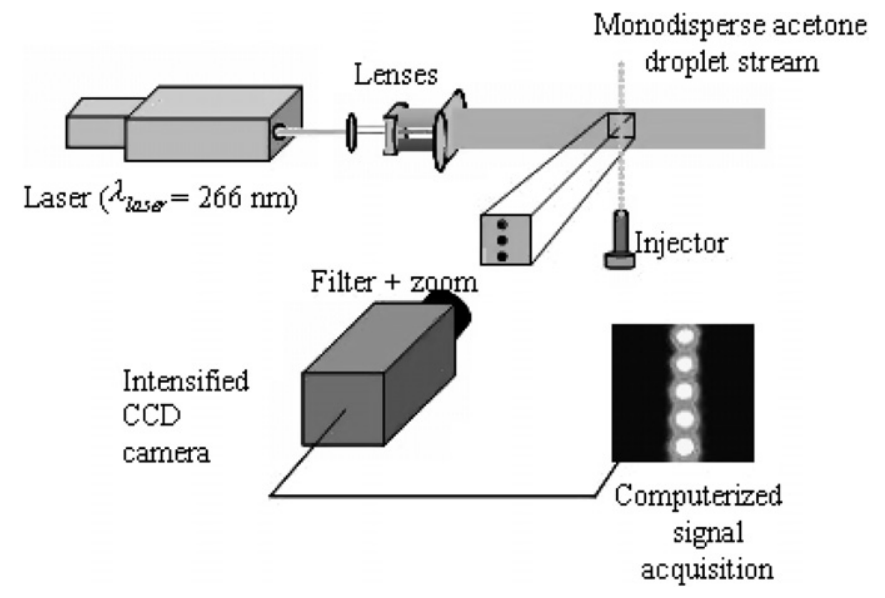

Fig. 3. Acetone PLIF experimental set-up. of the imaging lens to absorb the scattered light while transmitting the acetone fluorescence.

In the present experiments, performed under constant pressure conditions and with a moderate temperature evolution (less than $75^{\circ} \mathrm{C}$ ), the fluorescence signal emitted by acetone molecules is proportional to acetone concentration, which corresponds to fuel concentration in the surrounding gas phase in the case of vaporizing acetone droplets. In addition to classical corrections for background luminosity, non-uniformities in the collection optics and variations in the laser sheet profile, the correction introduced in [11] consists of reducing the influence of halation phenomenon on fuel vapor measurements. Mie scattering is first performed on a monodisperse stream of ethanol droplets because ethanol is a non-fluorescent fuel. Spatial extent of halation is determined from these Mie experiments. PLIF imaging on the same monodisperse droplet stream operating with acetone fuel is then carried out. Halation intensity from Mie experiments (normalized by the value of the LIF signal at the droplet surface) is subtracted from PLIF images. This corrected intensity is then converted into fuel vapor concentration around droplets via a calibration of fluorescence signals which is performed in a cell where temperature and acetone concentration are well controlled.

\section{Modeling of the liquid phase heat and mass transfer}

In the following modeling, the effect of the motion of the droplet boundary due to vaporization is neglected, which can be justified by assuming that the droplet surface regression rate is small for low evaporation regimes. The temperature $T$ within a droplet, taking into account the effect of the internal liquid circulation, is governed by the equation:

$\frac{\partial T^{*}}{\partial t^{*}}+\overrightarrow{V^{*}} \cdot \overrightarrow{\nabla^{*}} T^{*}=\frac{1}{P e} \Delta^{*} T^{*}$

where $\vec{V}$ is the liquid phase velocity vector, $P e$ is the droplet Peclet number, $P e=\frac{U_{s} R}{a_{1}}$ and $a_{1}$ is the thermal diffusivity of the liquid. In Eq. (5), the spatial coordinates are normalized by the droplet radius $R$ and the velocity by the maximum velocity $U_{\mathrm{S}}$ at the droplet surface. The nondimensional time is defined by $t^{*}=\frac{U_{\mathrm{S}}}{R} t$. The temperature $T$ can be normalized by using any reference because Eq. (5) is linear with $T$. It is assumed that the internal streamlines of the liquid circulating within the droplet follow a spherical Hill vortex pattern, according to the stream function $\psi[16]$ :

$\psi\left(r^{*}, \theta, \varphi\right)=-\frac{U_{\mathrm{s}} R^{2}}{2} r^{* 2}\left(1-r^{* 2}\right) \sin ^{2} \theta$

where $\left(r^{*}, \theta, \varphi\right)$ are the spherical coordinates of a point of the droplet, and $r^{*}=r / R$ is the non-dimensional radius. The maximum surface velocity $U_{\mathrm{S}}$ is given by $U_{\mathrm{S}}=$ $\frac{1}{6 \pi}\left(V_{\infty}-V_{\mathrm{d}}\right)\left(\frac{\mu_{\mathrm{g}}}{\mu_{\mathrm{l}}}\right) \operatorname{Re} C_{\mathrm{F}}[17]$, where $\mu_{\mathrm{g}}$ and $\mu_{1}$ are the dynamic viscosities of the gas and the liquid, $R e$ the droplet Reynolds number, $V_{\infty}$ the gas velocity far from the droplet 
and $V_{\mathrm{d}}$ the droplet velocity. $C_{\mathrm{F}}$ is the friction coefficient estimated, for the case of an isolated evaporating droplet, as:

$C_{\mathrm{F}}=\frac{12.69}{R e^{2 / 3}\left(1+B_{\mathrm{M}}\right)}$

where $B_{\mathrm{M}}$ is the mass transfer Spalding number. Since the droplet radius, the velocity $U_{\mathrm{s}}$ and the physical properties are time-varying, Eq. (5) is solved under the quasi-steady assumption, i.e. the evolution of droplet temperature will be seen as a succession of steady states. Therefore, Eq. (5) is solved at each time step with updated droplet radius, Peclet number and physical properties, in order to determine the next state. The liquid phase properties are estimated at the droplet average temperature. The physical properties of the gas phase are evaluated at the reference state $\left(T_{\text {ref }}\right.$ and $\left.Y_{\mathrm{K}, \text { ref }}\right)$ according to the " $1 / 3$ rule" [18], $T_{\text {ref }}=T_{\mathrm{S}}+$ $\left(T_{\infty}-T_{\mathrm{S}}\right) / 3$ and $Y_{\mathrm{K}, \text { ref }}=Y_{\mathrm{K}, \mathrm{S}}+\left(Y_{\mathrm{K}, \infty}-Y_{\mathrm{K}, \mathrm{S}}\right) / 3$. The fuel vapor mass fraction $Y_{\mathrm{K}, \mathrm{S}}$ at the droplet surface is calculated assuming the liquid-vapor equilibrium, by the ClausiusClapeyron law:

$P_{\mathrm{S}}\left(T_{\mathrm{S}}\right)=P\left(\frac{Y_{\mathrm{K}, \mathrm{S}}}{M_{\mathrm{K}}}\right) /\left(\frac{Y_{\mathrm{K}, \mathrm{S}}}{M_{\mathrm{K}}}+\frac{1-Y_{\mathrm{K}, \mathrm{S}}}{M_{\text {air }}}\right)$

where $P_{\mathrm{S}}\left(T_{\mathrm{S}}\right)$ is the vapor saturated pressure at the droplet surface temperature $T_{\mathrm{S}}, P$ the ambient pressure, $M_{\mathrm{K}}$ and $M_{\text {air }}$ the molecular weight of fuel and air respectively.

Eq. (5) is solved under the initial condition $T(r, t=0)=$ $T_{\text {inj }}$ (injection temperature of the liquid fuel) supplemented by the conditions at the droplet surface $2 \pi R^{2} \lambda_{1} \int_{\theta=0}^{\pi}\left(\frac{\partial T}{\partial r}\right)_{r=R} \sin \theta \mathrm{d} \theta=Q_{\mathrm{L}}(t)$, where $\lambda_{1}$ is the thermal diffusivity of the liquid and $Q_{\mathrm{L}}$ the heat flux penetrating into the droplet by conduction and convection from the gas phase. The instantaneous heat flux $Q_{\mathrm{L}}$ is evaluated by means of the overall energy budget equation, where radiative exchanges are neglected:

$Q_{\mathrm{L}}=\Phi_{\mathrm{C}}-\Phi_{\text {vap }}$

where $\Phi_{\mathrm{C}}=\frac{N u \lambda_{g} \pi D}{4}\left(T_{\infty}-T_{\mathrm{S}}\right)$ is the convective heat flux exchanged with the gaseous environment and the heat flux due to vaporization $\Phi_{\text {vap }}$ is defined by:

$\Phi_{\text {vap }}=L_{\mathrm{v}} \pi D \rho_{\mathrm{g}} D_{\mathrm{g}} B_{\mathrm{M}} S h$

where $L_{\mathrm{v}}$ is the latent heat of vaporization of the liquid fuel and $D_{\mathrm{g}}$ the molecular diffusivity of fuel vapor into air. The Nusselt number $N u$ and Sherwood number $S h$, according to the film theory, are expressed following the suggested forms of Abramzon and Sirignano [17]. In such a configuration, the influence of droplet-to-droplet interactions should be taken into account because the reduced distance parameter $C$ is less than 10. According to Sirignano [19], Hill's spherical vortex approximation remains valid in the case of interacting droplets. Droplet-to-droplet interactions should affect only heat, mass and momentum transfer by modifying the values of the Nusselt and Sherwood numbers and drag coefficient. It is well known that the decrease of the reduced droplet spacing leads to a decrease of the
Nusselt and Sherwood numbers [20]. Therefore, due to similarities between heat and mass transfer, it was shown that the interaction effects are similar on both Nusselt and Sherwood numbers, and these effects may be described by applying the same reduction factor $\eta(C)$ which depends only on the reduced droplet spacing $C[21]$ :

$\eta(C)=\frac{S h}{S h_{\text {iso }}}=\frac{N u}{N u_{\text {iso }}}$

where $N u_{\text {iso }}$ and $S h_{\text {iso }}$ represent the Nusselt and Sherwood numbers for isolated, moving, evaporating droplets defined by Abramzon and Sirignano [17]. The correlation suggested by Atthasit et al. [22], obtained for a pure evaporating droplet stream, is used:

$\eta(C)=1-0.57\left(\frac{1-\mathrm{e}^{-0.13(C-6)}}{1+\mathrm{e}^{-0.13(C-6)}}\right), \quad(2.5 \leqslant C \leqslant 16)$

The resolution of Eq. (5) is performed by splitting the nondimensional temperature on the Legendre polynomials based at each time step [23]. As proposed by Abramzon and Sirignano [17], equation solving takes into account the regulation mechanism which correlates the penetrating heat flux $Q_{\mathrm{L}}$, droplet surface temperature, fuel vapor mass fraction at the droplet surface $Y_{\mathrm{K}, \mathrm{S}}$ and the vaporization rate $\dot{m}$.

\section{Numerical simulation of the gas phase}

\subsection{Governing equations}

The conservation equations for momentum, mass and enthalpy in the gas phase, for a non-reactive medium may be written as follows:

$\rho_{\mathrm{g}}\left(\frac{\partial \vec{V}}{\partial t}+\vec{V} \overline{\bar{\nabla}} \vec{V}\right)=-\overrightarrow{\nabla P}+\vec{\nabla} \cdot\left(\frac{\mu_{\mathrm{g}}}{2}\left(\overline{\bar{\nabla}} \vec{V}+{ }^{t} \overline{\bar{\nabla}} \vec{V}\right)\right)+\rho_{\mathrm{g}} \vec{g}$

$\frac{\partial \rho_{\mathrm{g}}}{\partial t}+\vec{\nabla} \cdot\left(\rho_{\mathrm{g}} \vec{V}\right)=0$

$\frac{\partial \rho_{\mathrm{g}} Y_{\mathrm{K}}}{\partial t}+\vec{\nabla} \cdot\left(\rho_{\mathrm{g}} Y_{\mathrm{K}} \vec{V}\right)+\vec{\nabla} \cdot\left(-D_{\mathrm{K}} \vec{\nabla}\left(\rho_{\mathrm{g}} Y_{\mathrm{K}}\right)\right)=0$

$\frac{\partial\left(\rho_{\mathrm{g}} C_{p_{\mathrm{g}}} T\right)}{\partial t}+\vec{\nabla} \cdot\left(\rho_{\mathrm{g}} C_{p_{\mathrm{g}}} T \vec{V}\right)-\vec{\nabla} \cdot\left(\lambda_{\mathrm{g}} \vec{\nabla}(T)\right)=0$

These equations are strongly coupled, mainly because of the heat and mass transport by the gas flow. In the present computations, the dependence of gas physical properties on temperature and vapor concentration is taken into account. In the case of a binary mixture, the specific density of the (fuel + air) mixture $\rho_{\mathrm{g}}$ and the heat capacity $C_{p_{\mathrm{g}}}$ of the mixture are given by:

$\rho_{\mathrm{g}}\left(T, Y_{\mathrm{K}}\right)=\left(Y_{\mathrm{K}} / \rho_{\mathrm{Kg}}(T)+\left(1-Y_{\mathrm{K}}\right) / \rho_{\text {air }}(T)\right)^{-1}$

$C_{p_{\mathrm{g}}}\left(T, Y_{\mathrm{K}}\right)=Y_{\mathrm{K}} C_{p_{\mathrm{Kg}}}(T)+\left(1-Y_{\mathrm{K}}\right) C_{p_{\text {air }}}(T)$

Evaluation of the viscosity $\mu_{\mathrm{g}}$ and the thermal conductivity $\lambda_{\mathrm{g}}$ of a binary mixture can be estimated according to the Wilke relationships [24]. 


\subsection{Solving assumption}

Resolution of equations is performed with assumptions specific to the case of the monodisperse droplet stream, which consists of a quasi-periodical linear arrangement of droplets. Therefore, the following hypotheses are made:

- the cylindrical coordinates system $(r, \theta, z)$, with $z$ corresponding to the jet axis, is used,

- because of the cylindrical symmetry around the stream axis, velocity, temperature and fuel vapor concentration fields are invariant according to the coordinate $\theta$,

- droplets are supposed to be arranged periodically, making it possible to solve the equations on a portion of the stream restricted to a length corresponding to the droplet spacing. A sketch of the elementary cell where equations are solved is presented in Fig. 4. Therefore, the resulting two-dimensional equations can be solved using an Eulerian approach. With such a space limitation of the computational domain, a fine description of the temperature, concentration and velocity fields around droplets can be achieved without being time-consuming.

Droplet surface temperature is determined according to the model of the liquid phase and it is supposed to be spatially uniform but time-varying. Furthermore, in such a low evaporation regime at moderate ambient temperature, the effect of the droplet surface regression and Stefan flow on the fuel vapor field is neglected.

The hypothesis of periodic approximation consists of calculating the temporal evolution of the boundary layers around a droplet using an Eulerian approach. In particular, while in the experiments droplets are moving in a still environment with zero velocity, in the simulations the droplet is motionless in a flow of axial bulk velocity equal to the experimental value of droplet velocity. As a consequence of the periodicity, there is no stationary solution of the equations. After a sufficiently long time, the distance covered by the droplet becomes large compared to the droplet spacing, which permits the assumption that droplet-to-droplet interactions are well-established. Comparison is then possible with the case of a semi-infinite droplet stream in a steady regime. Using this approach,

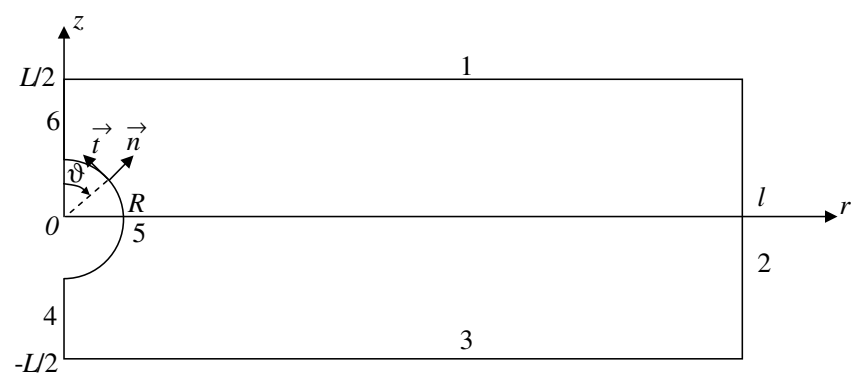

Fig. 4. Sketch of the elementary domain where gas phase equations are solved. the droplet spacing is constant with time, whereas, the droplet size reduction due to evaporation and the drag force tend actually to reduce this spacing. However, although the gas phase pattern is calculated for a fixed droplet spacing in the solving of the diffusion/convection equation of vapor, droplet surface temperature, obtained in the simulation of the liquid phase, takes into account this reduction of droplet velocity. Nonetheless, this approximation for the calculation of the gas phase is likely to weakly affect the results.

\subsection{Initial and boundary conditions}

At $t=0$, at each point of the computational domain, temperature, fuel mass fraction and velocity of the gas phase are initially equal to their value far from the droplet stream. The resolution domain exhibits 6 boundaries (Fig. 4).

The conditions imposed on boundaries 1 and 3 are similar in order to respect the condition of periodicity. Boundaries 1 and 3 are symmetry planes for the radial component of the velocity $U$, leading to $\left.\frac{\partial U}{\partial z}\right|_{z= \pm L / 2}=0$, where $L$ is the droplet spacing (center-to-center).

The conditions of periodicity are given by:

$\left\{\begin{array}{l}A(r, z=+L / 2)=A(r, z=-L / 2) \\ \left.\frac{\partial A}{\partial z}\right|_{z=+L / 2}=\left.\frac{\partial A}{\partial z}\right|_{z=-L / 2}\end{array}, \quad \forall r>0\right.$

$A$ is either the axial component of the gas velocity $\vec{V}$, the temperature $T$, the pressure $P$ or the fuel mass fraction in the gas phase $Y_{\mathrm{K}}$.

At boundary 2 , the conditions of the gas phase far from the droplet stream are written as:

$A(r=l, z)=A_{\infty}, \quad A=T, Y_{\mathrm{K}}, P, U, V$

where $l$ represents the extend of the calculation domain (Fig. 4).

Since the present approach considers motionless droplets, the gas phase velocity field far from the droplet stream is given by:

$$
\left\{\begin{array}{l}
U_{\infty}=0 \\
U_{\infty}=-V_{\mathrm{d}}
\end{array}\right.
$$

where $V_{\mathrm{d}}=V_{\mathrm{inj}}$ is given in Table 1 for all the conditions investigated.

Additionally, the variables $T, Y_{\mathrm{K}}, U, V$ and $P$ must tend asymptotically to their value in $r=l$ :

Table 1

Summary of the aerothermal conditions of the different test cases

\begin{tabular}{llllllll}
\hline & Case 1 & Case 2 & Case 3 & Case 4 & Case 5 & Case 6 & Case 7 \\
\hline$D(\mu \mathrm{m})$ & 144 & 170 & 130 & 230 & 136 & 164 & 193 \\
$V_{\text {inj }}(\mathrm{m} / \mathrm{s})$ & 9.5 & 9 & 9.5 & 9.5 & 9.5 & 9.5 & 9.5 \\
$C$ & 4.5 & 5.2 & 4.5 & 3.5 & 4 & 5.7 & 8 \\
$\eta(C)$ & 0.37 & 0.4 & 0.37 & 0.34 & 0.35 & 0.42 & 0.5 \\
$T_{\text {inj }}\left({ }^{\circ} \mathrm{C}\right)$ & 39 & 39 & 25 & 25 & 27 & 27 & 27 \\
$T_{\text {air }}\left({ }^{\circ} \mathrm{C}\right)$ & 21 & 21 & 21 & 21 & 75 & 75 & 75 \\
\hline
\end{tabular}




$$
\left.\frac{\partial A}{\partial r}\right|_{r=1}=0, \quad A=T, Y_{\mathrm{K}}, P, U, V
$$

At boundaries 4 and 6 , which belong to revolution axis of the droplet stream, the condition is:

$$
\left.\frac{\partial A}{\partial r}\right|_{r=0}=0
$$

Boundary 5 corresponds to the droplet surface. As mentioned previously, in order to respect the assumption of the model for the liquid phase, the temperature is uniformly distributed at the droplet surface, but time-varying. The fuel mass fraction at the droplet surface is directly related to the surface temperature by assuming the liquidvapor equilibrium. This mass fraction can be calculated according to the Clausius-Clapeyron law (Eq. (8)).

For the velocity, the conditions at the droplet surface can be written as follows:

$$
\left\{\begin{array}{l}
\vec{V} \cdot \vec{n} \simeq 0 \\
\vec{V} \cdot \vec{t}=-U_{\mathrm{S}} \sin \theta
\end{array}\right.
$$

where the vectors $\vec{n}$ and $\vec{t}$ are respectively the normal and the tangential vectors to the droplet surface (Fig. 4).

\subsection{Numerical resolution}

The resolution of the equations was performed using the finite elements method by the commercial code FEM$\mathrm{LAB}^{\mathrm{TM}}$. The mesh consists of about 8000 elements and is relatively dense near the surface of the droplet and in the vicinity of boundaries 1 and 3 where the conditions of periodicity apply.

\section{Results}

The different test cases detailed in this section correspond to conditions achievable by best tuning the frequency of the monodisperse injector, knowing that the operating frequency, droplet diameter and droplet spacing are coupled altogether.

The first experiments (cases 1 and 2 in Table 1) consist of injecting overheated acetone droplets $\left(T_{\text {inj }}=39^{\circ} \mathrm{C}\right)$ in a free and quiescent atmosphere at ambient temperature $\left(T_{\text {air }}=21^{\circ} \mathrm{C}\right)$ and atmospheric pressure. Two different droplet diameters and spacing are considered $(D=$ $144 \mu \mathrm{m}, C=4.5$ and $D=170 \mu \mathrm{m}, C=5.0$ ). The evolution of droplet mean temperature as a function of time is depicted in Fig. 5. It is characterized by a strong temperature decrease in the break-up phase of the liquid jet (not shown), followed by a smoother decrease due to the dominating vaporization phenomena together with heat transfer by forced convection. The temperature evolution of these two droplet streams is quite comparable, which can be qualitatively explained by the occurrence of two opposite phenomena: on one hand, large droplets (case 2) tend to cool down slower than small ones (case 1) and on the other hand, large droplets have the highest distance parameter which enhances vaporization and the subsequent cooling. The simulation results, i.e. droplet mean temperature and droplet surface temperature, are also reported in Fig. 5. It is important to notice that since heat and mass transfer is not modeled in the break-up region of the liquid jet, the simulation of the liquid phase is started at the first measurement point $(z=10 \mathrm{~mm}$ downstream from the injector). The starting temperature in the calculations is equal to the injection temperature, uniformly

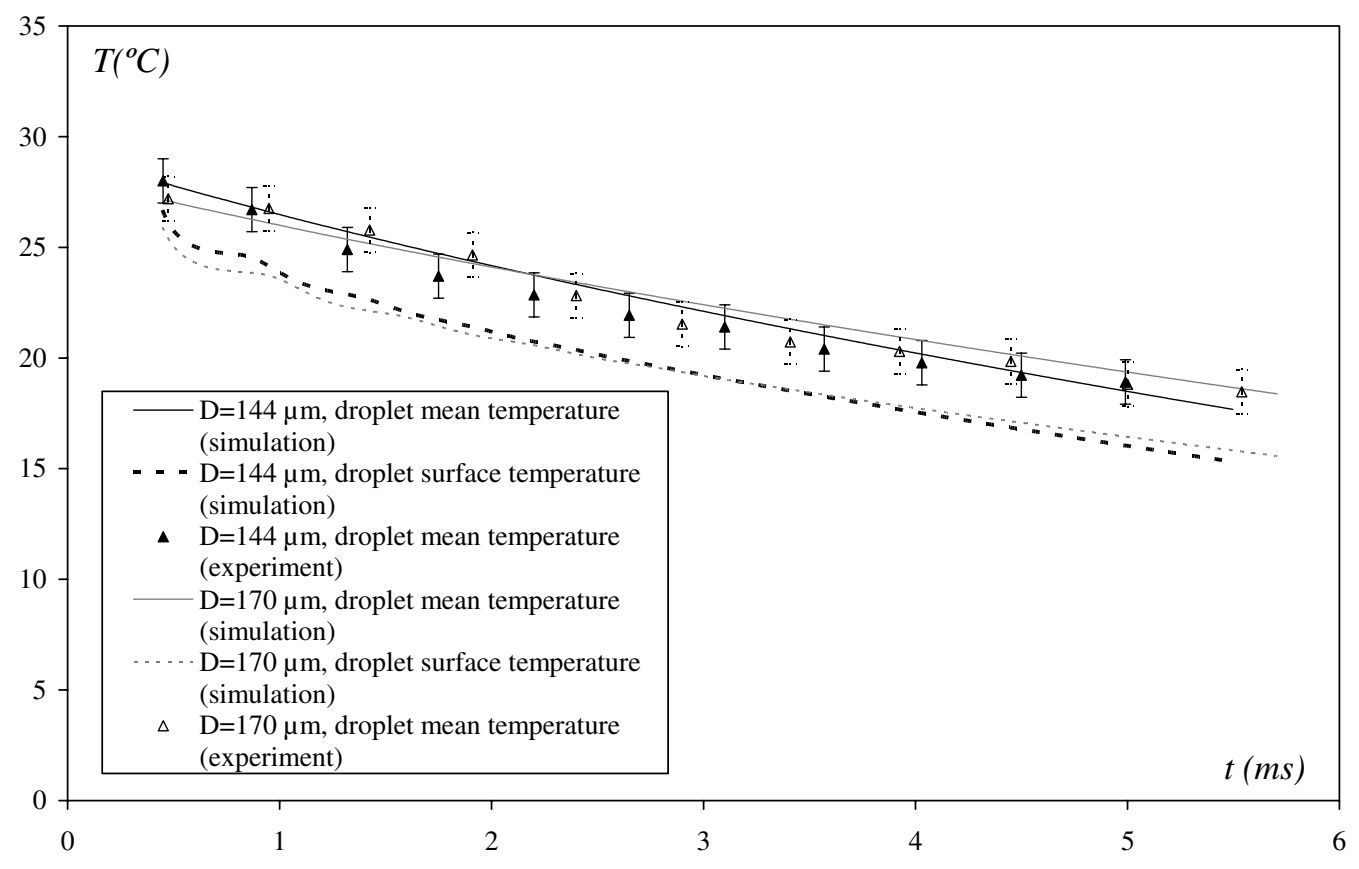

Fig. 5. Experimental and calculation results of the temporal evolution of droplet temperature for $D=144 \mu \mathrm{m}$ and $D=170 \mu \mathrm{m}$. 
distributed over the droplet volume. The artifact on the evolution of the surface temperature at $t<1 \mathrm{~ms}$ is due to the fixed uniform initial conditions. Nonetheless, for $t>$ $1 \mathrm{~ms}$, the calculated mean temperature shows good agreement with the experimental values and lies within the error bars $( \pm 1 \mathrm{~K})$ which represent the uncertainty of the 2-color LIF technique. In particular, the decay rate of droplet mean temperature is well described by the simulation.

Using the numerical values of surface temperature $T_{\mathrm{S}}$ and surface velocity $U_{\mathrm{S}}$ obtained from Fig. 5 as inputs, fuel vapor spatial distribution in the gas phase around the droplet stream can be calculated. Fig. 6 displays a typical example of such distribution for a droplet diameter of $D=144 \mu \mathrm{m}$ and droplet spacing of 4.5. On the zoomed picture of Fig. 6, the asymmetric fuel vapor repartition around the droplet is clearly visible. In particular, the droplet wake and the accumulation of vapor in the recirculation zone behind the droplet (i.e. on the droplet side opposed to the bulk flow direction) are well captured by the simulation. Radial profiles of acetone vapor molar fraction around a droplet can be derived from Fig. 6. Fig. 7 shows comparison between experimental and numerical radial profiles at different axial locations $(z=20 \mathrm{~mm}, 28 \mathrm{~mm}$, $36 \mathrm{~mm}$ and $44 \mathrm{~mm}$ ) for $D=144 \mu \mathrm{m} D=170 \mu \mathrm{m}$ and for the same initial conditions as in Fig. 5. The vertical error bars represent the rms of fluctuations of fuel vapor molar fraction around the mean value. The horizontal error bars represent the uncertainty on the measurement location resulting from the spatial resolution of the technique $(16 \mu \mathrm{m}$ per pixel). For clarity, the horizontal error bars are displayed only for the first point of each profile in Fig. 7. However, each point of the profiles is obviously associated with the same uncertainty on its location. Except for the position $z=20 \mathrm{~mm}$, where a significant difference between numerical results and experimental data is observed, a good agreement between experiment and simu-

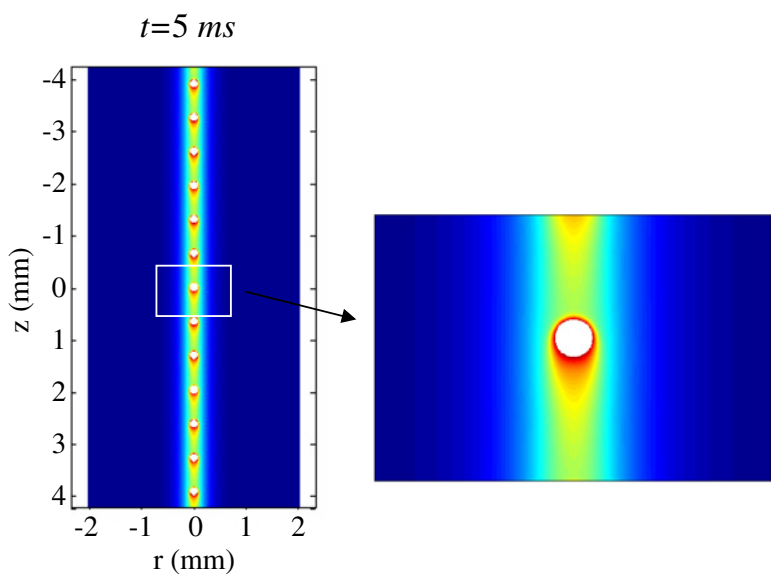

$\chi(\%)$

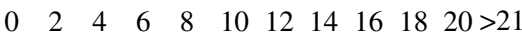

Fig. 6. Simulation of the spatial distribution of acetone vapor molar fraction around droplets at $z=44 \mathrm{~mm}(t=5 \mathrm{~ms})$ for $D=144 \mu \mathrm{m}$. lation can be observed. In particular, the radial decrease of molar fraction is properly modeled with the simulation, which generates confidence that the hypotheses made for the calculation of both phases were appropriate. The discrepancy at $z=20 \mathrm{~mm}$ can be attributed to the insufficient convergence of the Eulerian calculation of the gas phase because the equivalent number of droplets launched from the injection point $N$ is probably too low.

The second experiment concerns the specific effect of droplet diameter on mass transfer between the liquid and gas phases. Droplets with strongly different diameters $(D=130 \mu \mathrm{m}$ and $D=230 \mu \mathrm{m}$, cases 3 and 4 in Table 1$)$, but with similar distance parameter $(C=4.5$ and $C=3.5$ respectively) are considered for $T_{\text {inj }}=25^{\circ} \mathrm{C}$ and $T_{\text {air }}=$ $21^{\circ} \mathrm{C}$. Both experimental and calculation results obtained for the temperature of the liquid phase are shown in Fig. 8. A reasonable agreement between experiment and simulation is observed for both diameters, although droplet mean temperature obtained with calculation is slightly underestimated at large times. As expected, droplet temperature decreases faster for the smallest droplet diameter. Discrepancies observed may result from different causes. For example, it is well known that the thermodynamic properties of liquid fuel or vapor can be a major source of uncertainty for droplet evaporation calculations $[17,20]$. In addition, the friction coefficient considered in the present calculation is that of an isolated droplet which probably differs from that of a droplet in a monodisperse stream. Unfortunately, no information on that subject is available in the literature and, therefore, this approximation were used. Finally, the expression of $\eta(C)$ in Eq. (12) is determined experimentally with an uncertainty of about $20 \%$ and this may partly explain the differences observed between experiments and simulations in Fig. 8. Experimental radial profiles of fuel vapor molar fraction around a droplet at $t=3 \mathrm{~ms}$ after injection (about $z=30 \mathrm{~mm}$ ) are compared with calculation in Fig. 9. For both droplet diameters investigated, a good agreement between experimental and numerical data can be noticed, both in terms of the decay rate of molar fraction and in terms of its spatial extent. However, in many situations, it is often interesting to compare non-dimensional profiles, for example to understand the influence of a specific parameter. In the present case, it is believed that the fuel vapor boundary layer is a key point for the spatial distribution of fuel vapor around the droplet stream. The fuel vapor boundary layer thickness is estimated by simple dimensional analysis. The mass conservation at the droplet surface is written as:

$\frac{\Phi_{\text {vap }}}{L_{v}} Y_{\mathrm{S}}-\left.\pi D^{2} \rho_{\mathrm{g}} D_{\mathrm{g}} \frac{\mathrm{d} Y}{\mathrm{~d} r}\right|_{\mathrm{S}}=\frac{\Phi_{\text {vap }}}{L_{\mathrm{v}}}$

The vapor mass fraction gradient in the gas phase, $\left.\frac{\mathrm{d} Y}{\mathrm{~d} r}\right|_{\mathrm{S}}=-\frac{\Phi_{\mathrm{vap}}}{L_{\mathrm{v}}} \frac{\left(1-Y_{\mathrm{S}}\right)}{\pi D^{2} \rho_{\mathrm{g}} D_{\mathrm{g}}}$, can be estimated by:

$\left.\frac{\mathrm{d} Y}{\mathrm{~d} r}\right|_{\mathrm{S}} \sim \frac{Y_{\mathrm{S}}-Y_{\infty}}{\delta} \sim \frac{\Phi_{\text {vap }}}{L_{\mathrm{v}}} \frac{\left(1-Y_{\mathrm{S}}\right)}{\pi D^{2} \rho_{\mathrm{g}} D_{\mathrm{g}}}$ 
$\chi(\%)$

a.1) $D=144 \mu \mathrm{m}, t=2.2 \mathrm{~ms}, \mathrm{~N}=36$

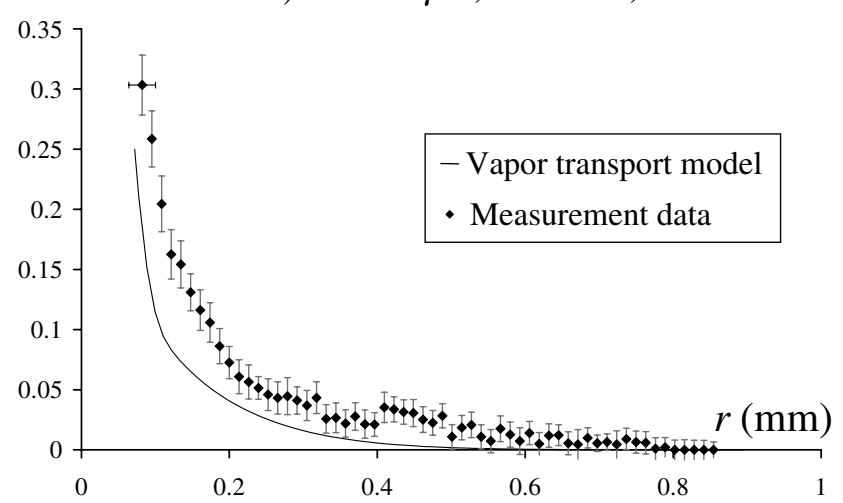

$\chi(\%) \quad$ a.2) $D=144 \mu \mathrm{m}, t=3.1 \mathrm{~ms}, N=52$
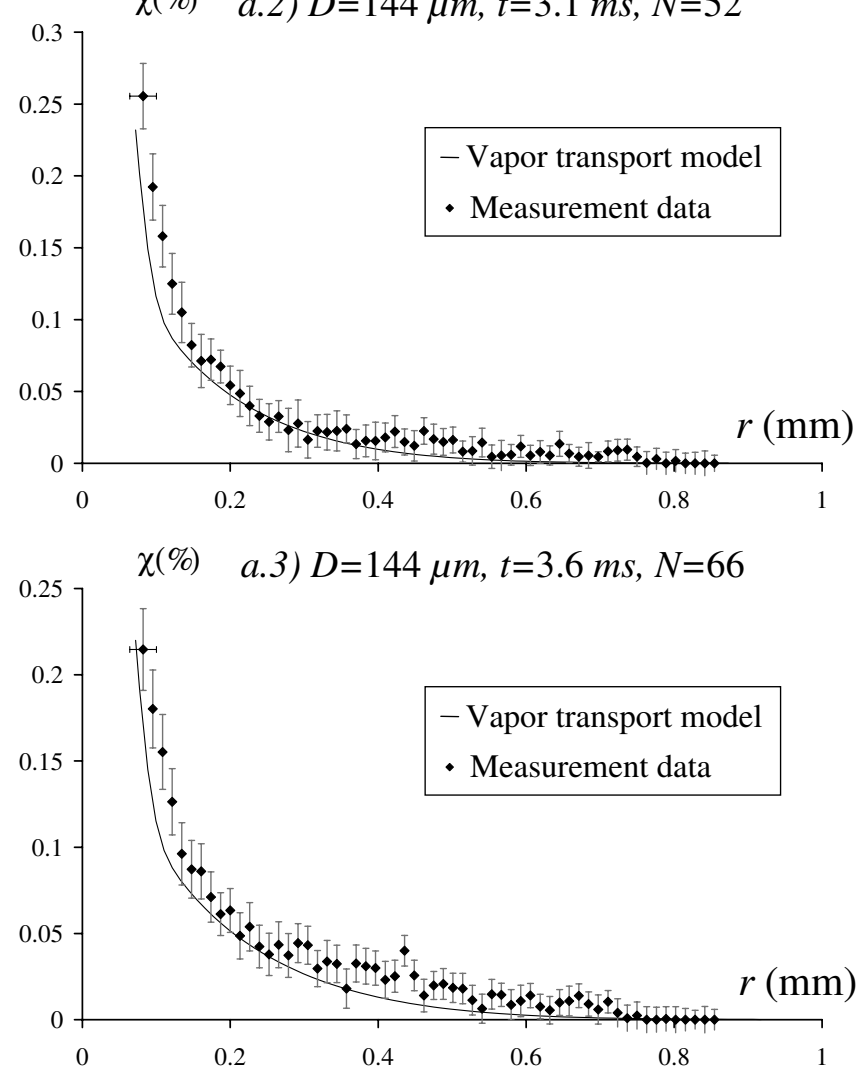

$\chi(\%)$ a.4) $D=144 \mu \mathrm{m}, t=5 \mathrm{~ms}, N=81$

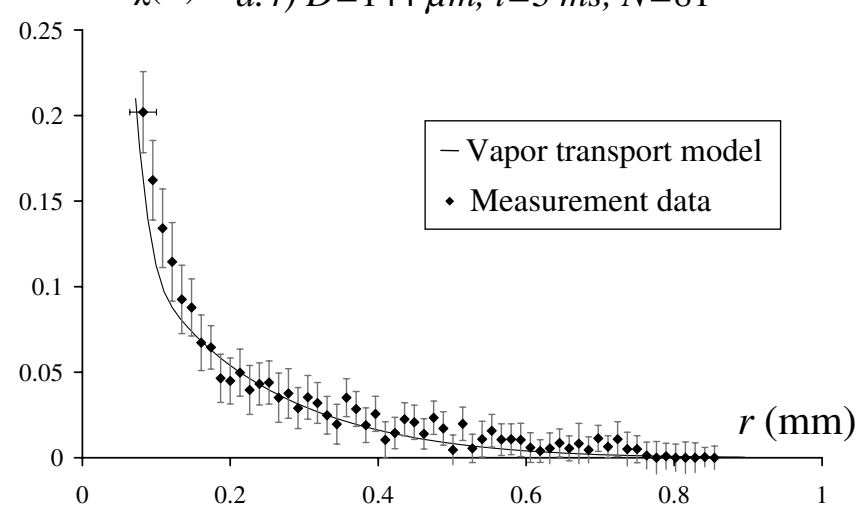

$\chi(\%) \quad b .1) D=170 \mu m, t=2.4 \mathrm{~ms}, N=23$

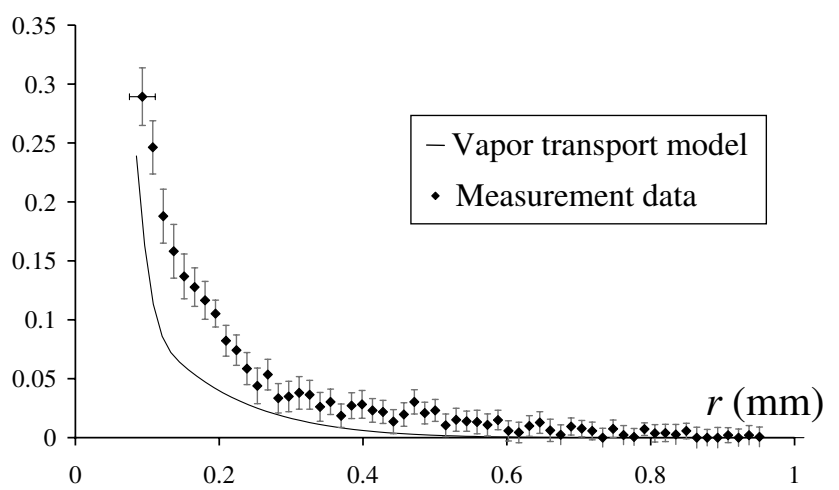

$\chi(\%)$

b.2) $D=170 \mu \mathrm{m}, t=3.4 \mathrm{~ms}, N=31$

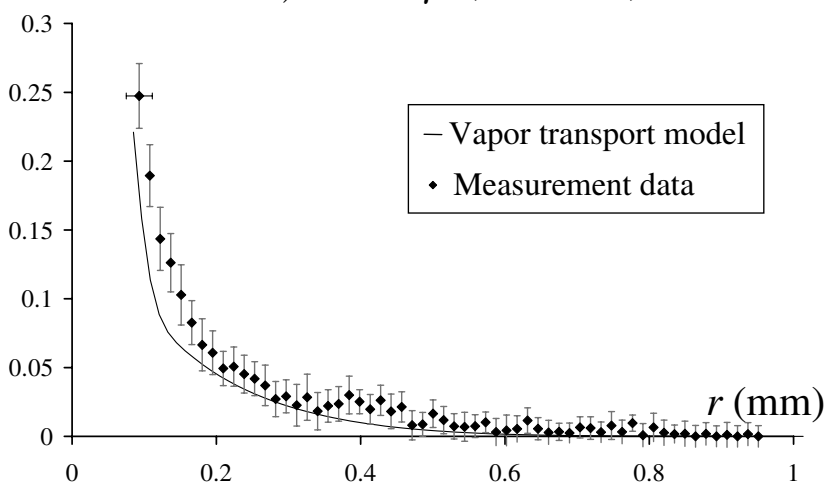

$\chi(\%) \quad b .3) D=170 \mu \mathrm{m}, t=4.5 \mathrm{~ms}, N=41$

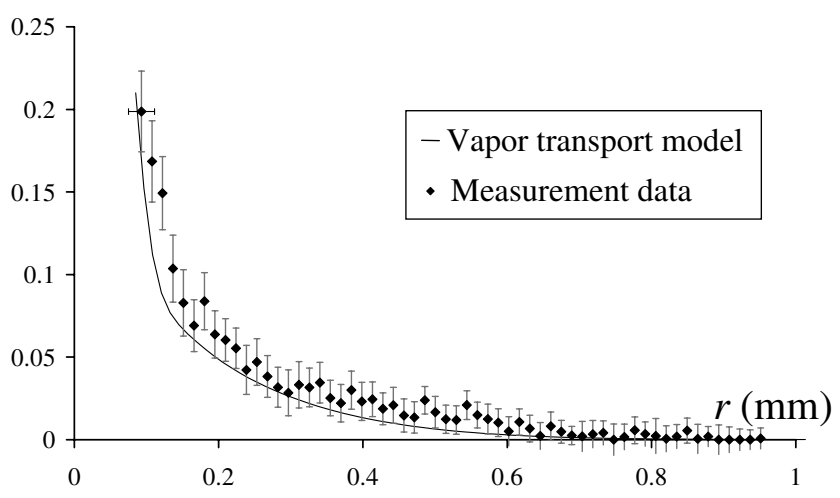

$\chi(\%) \quad b .4) D=170 \mu m, t=5.5 \mathrm{~ms}, N=50$

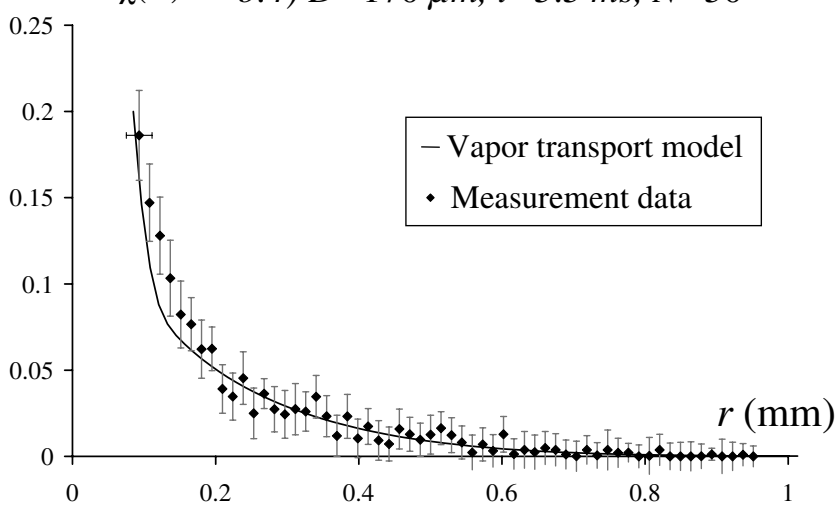

Fig. 7. Radial profiles of acetone vapor molar fraction around a droplet at $z=20 \mathrm{~mm}, 28 \mathrm{~mm}, 36 \mathrm{~mm}$ and $44 \mathrm{~mm}$, for $D=144 \mu \mathrm{m}$ (a) and $D=170 \mu \mathrm{m}$ (b). Comparison with numerical simulations. 


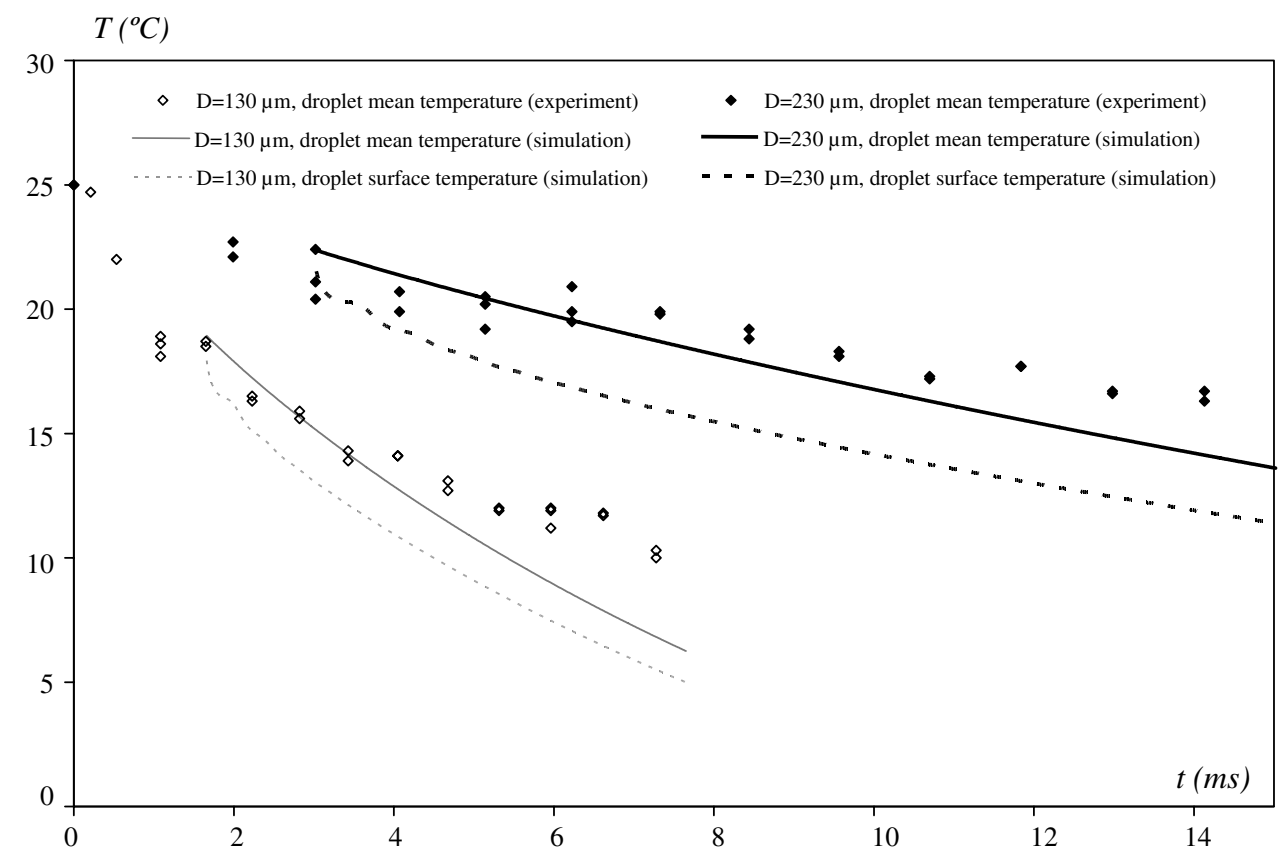

Fig. 8. Experimental and calculation results of the time evolution of droplet temperature for $D=130 \mu \mathrm{m}$ and $D=230 \mu \mathrm{m}$.

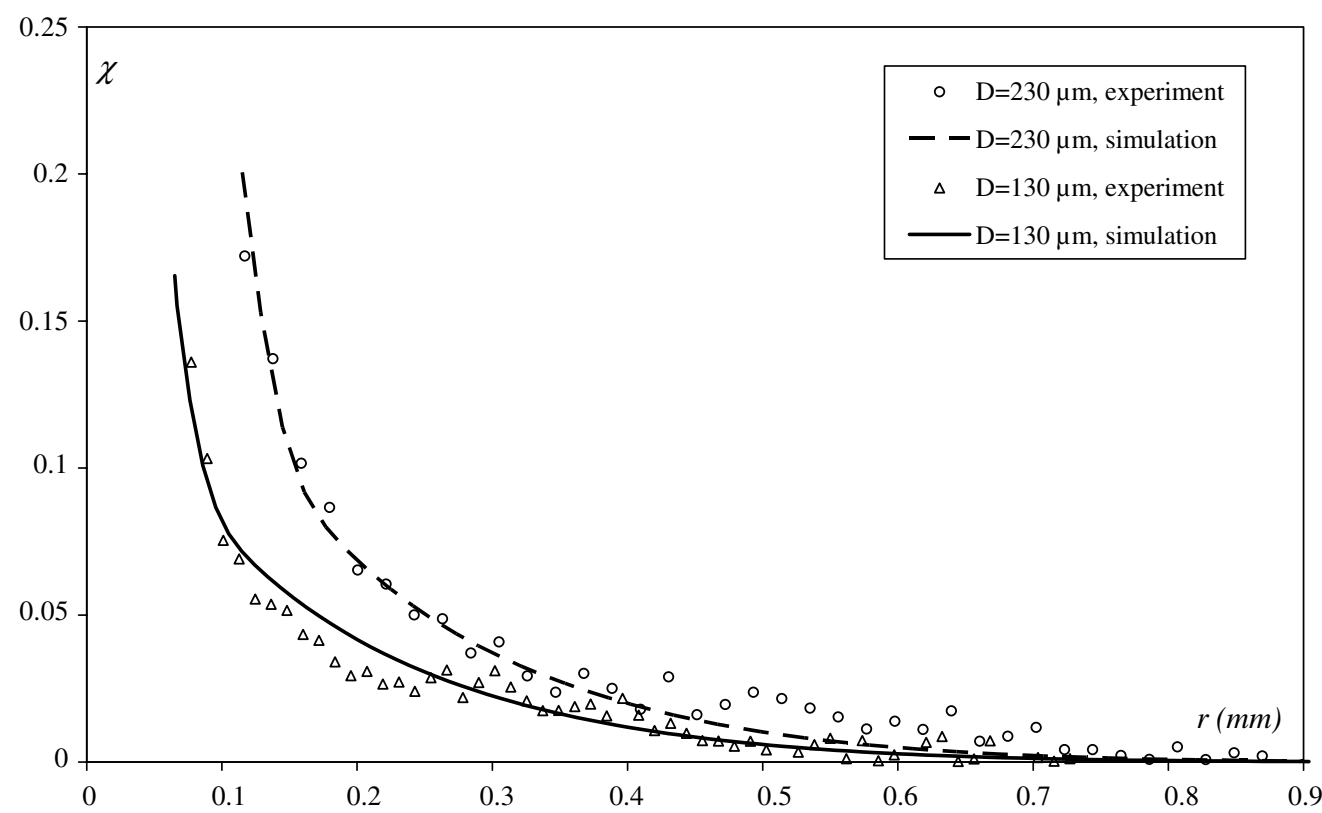

Fig. 9. Radial profiles of fuel vapor molar fraction around a droplet at $t=3 \mathrm{~ms}$ after injection for $D=130 \mu \mathrm{m}$ and $D=230 \mu \mathrm{m}$. Comparison with numerical results.

Using the expression of the vaporization flux (Eq. (10)), and the definition of the Spalding number $B_{\mathrm{M}}$, the vapor boundary layer thickness $\delta$ can be estimated by:

$\delta \sim \frac{D}{S h}=\frac{D}{\eta(C) S h_{\text {iso }}}$

where the expression $S h_{\text {iso }}$ of the Sherwood number for a moving isolated suggested in [17] is used. According to Eq. (27), the thickness of the boundary layer increases with droplet diameter. This behavior is clearly observed in the vapor molar fraction profiles of Fig. 9, where the profile corresponding to the largest diameter is broader. The validity of Eq. (27) is also checked more carefully by normalizing the radial distance by $\delta$ and the vapor molar fraction by its theoretical value at the droplet surface (Fig. 10). The correct overlap of both normalized experimental profiles confirms the suitability of the previously estimated thickness $\delta$ of the mass transfer boundary layer regarding the influence of droplet size. A similar conclusion applies also for both simulated profiles reported on the same figure. 


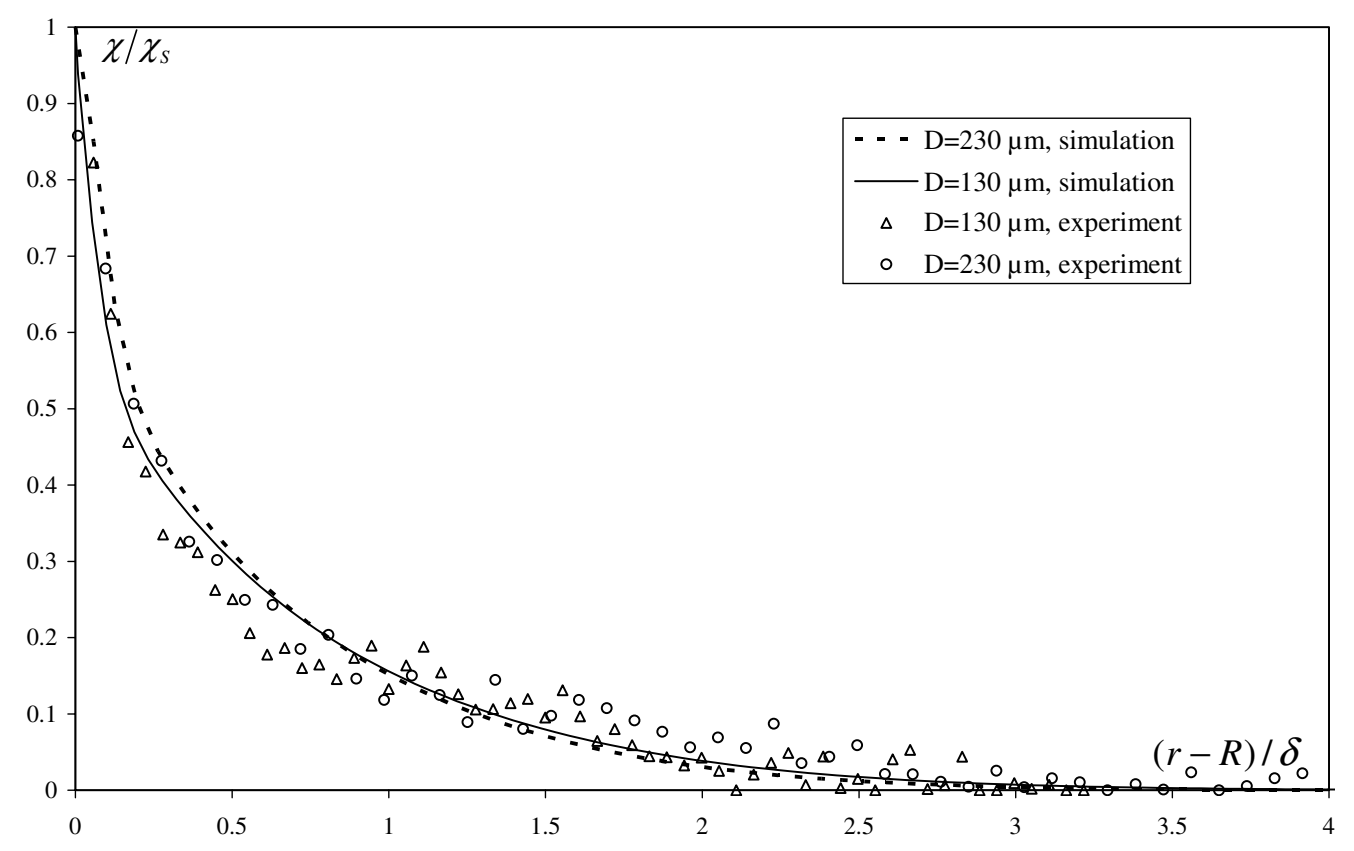

Fig. 10. Normalized radial profiles of fuel vapor molar fraction around a droplet at $t=3 \mathrm{~ms}$ after injection for $D=130 \mu \mathrm{m}$ and $D=230 \mu \mathrm{m}$. Comparison with simulations.

Finally, the influence of droplet spacing on fuel vapor distribution is investigated on three test cases (cases 5, 6 and 7 in Table 1). All these experiments performed in a large preheated enclosure, where air temperature is maintained at $75^{\circ} \mathrm{C}$ in order to enhance fuel vaporization. The frequency of the piezoceramic is adjusted so as to obtain the three different distance parameters summarized in Table 1. Radial profiles of vapor molar fraction are presented in Fig. 11, at the same time after injection $(t=4.66 \mathrm{~ms})$ for the three test cases with a normalization similar to that in Fig. 10. As can be seen in Fig. 11, the three vapor molar fraction profiles, corresponding to the different droplet spacing, are overlapping. Therefore, it is clear that the spatial distribution of vapor around a droplet can be described by two parameters:

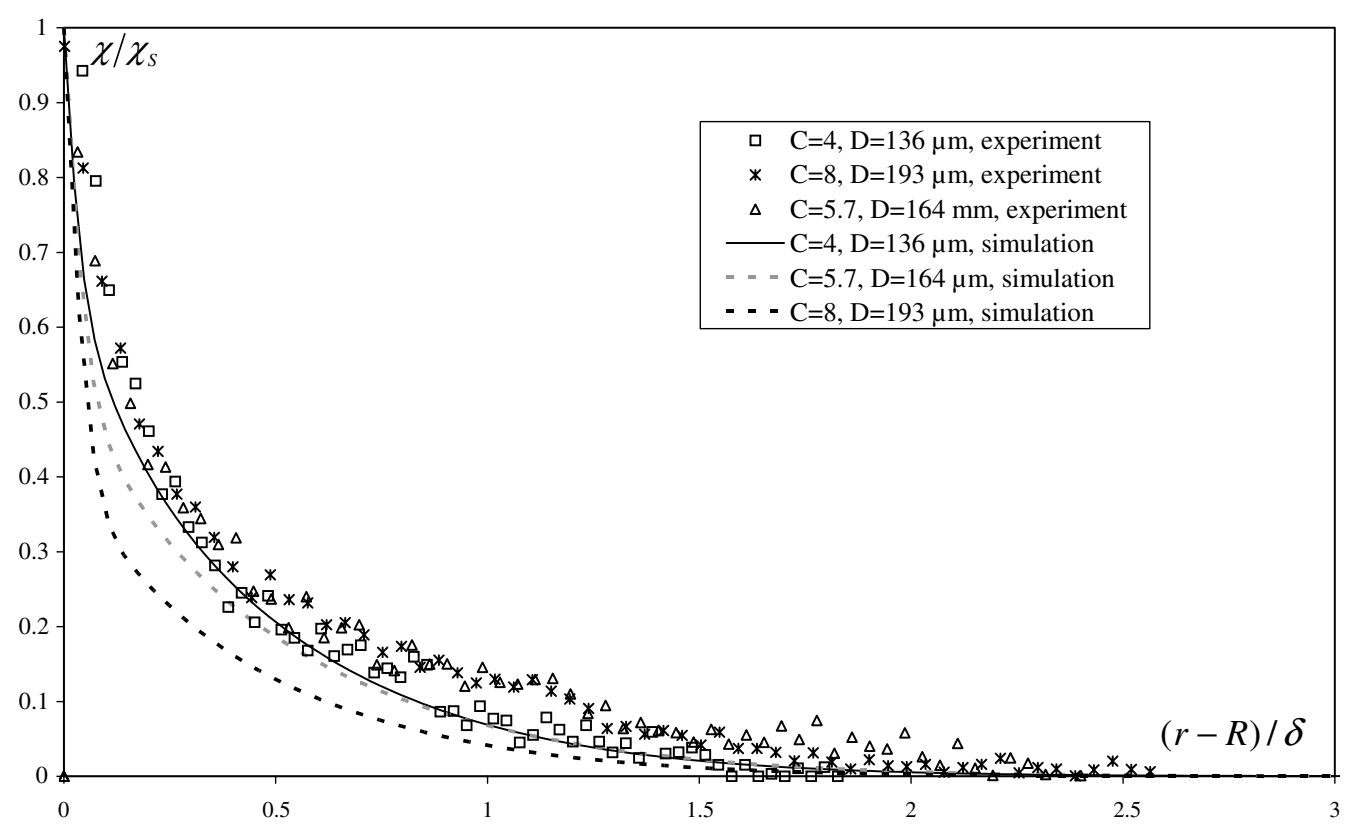

Fig. 11. Normalized fuel vapor molar fraction at $t=4.66 \mathrm{~ms}$ for $D=136 \mu \mathrm{m}(C=4), D=164 \mu \mathrm{m}(C=5.7), D=193 \mu \mathrm{m}(C=8)$. Comparison with numerical results. 
Table 2

Droplet temperature at $t=4.66 \mathrm{~ms}$ for the different test cases

\begin{tabular}{llll}
\hline At $t=4.66 \mathrm{~ms}$ & $T_{\mathrm{m}}$ measured & $T_{\mathrm{m}}$ calculated & $T_{\mathrm{s}}$ calculated \\
\hline Case 5 & 12.6 & 12.2 & 11.3 \\
$D=136 \mu \mathrm{m}$ & & & \\
$C=4$ & & 11.6 & 10.5 \\
Case 6 & 11.6 & & \\
$D=164 \mu \mathrm{m}$ & & 12.1 \\
$C=5.7$ & & \\
Case 7 & 13.6 & 13.5 & \\
$D=193 \mu \mathrm{m}$ & & & \\
$C=8$ & & & \\
\hline
\end{tabular}

- the boundary layer thickness $\delta$, where the factor $\eta(C)$ taking into account the interaction phenomena (Eq. (27)), is included.

- the vapor molar fraction at the droplet surface that can be well predicted from the simplified model presented in Section 4.

The calculated and measured droplet mean temperatures, as well as the calculated surface temperature, are summarized in Table 2, for the three investigated cases, at $t=4.66 \mathrm{~ms}$. Similarly to results in Figs. 5 and 8, experimental and numerical data are very close. Gas phase calculations are also reported in Fig. 11: a good agreement between experiment and calculation is noticed for the smallest distance parameter $(C=4)$. Some discrepancy is yet observed for $C=5.7$. Important difference is found for the largest distance parameter $(C=8)$. This disagreement can be attributed to the lack of convergence of the calculation at the considered time $(t=4.66 \mathrm{~ms})$. Indeed, for the largest distance parameter $(C=8)$, the equivalent number of launched droplets $N$ is only 27 whereas it is about 77 for $C=4$.

\section{Conclusion}

Combined measurements of the liquid phase temperature and fuel vapor distribution in a monodisperse stream of evaporating acetone droplets have been reported. Experimental results have been compared to numerical simulations of both the liquid and gas phases. The modeling of the liquid phase takes into account the internal fluid circulation and the influence of droplet-to-droplet interactions by modifying both Sherwood and Nusselt numbers with a factor depending only on the distance parameter. The gas phase simulation is based on an Eulerian approach of droplets periodically arranged in space. The coupling between both phases is realized by taking as a boundary condition for the gas phase simulation, the time-varying droplet surface temperature issued from the liquid phase calculation.

For moderate distance parameters, both liquid and gas phase measurements are in good agreement with calculations because the convergence is reached more rapidly. Analysis of the influence of the sole droplet diameter has shown that the mass transport boundary layer thickness can be safely approximated by $\delta \sim \frac{D}{S h}$, where the Sherwood number is calculated with the film theory as suggested by Abramzon and Sirignano [17]. The effect of droplet spacing, which characterizes droplet-to-droplet interactions, has also been investigated. Normalization of the radial distance by $\delta$ and the fuel vapor molar fraction by its value at the droplet surface leads to overlapping fuel vapor radial profiles for different droplet diameters and spacing. Consequently, droplet-to-droplet interactions in the range of investigation $(4 \leqslant C \leqslant 8)$ can be properly described by modifying both Nusselt and Sherwood numbers with a factor depending only on the distance parameter $C$, which modifies both conditions at the droplet surface and mass transport boundary layer thickness.

Further investigation will concern the extension of the range of droplet spacing, which requires a different strategy for droplet generation. Finally, droplet evaporation at high temperature needs to be investigated, both experimentally and numerically.

\section{Acknowledgement}

This work is supported by the European Community in the framework of the MUSCLES contract, Growth project GRD1-2001-40198.

\section{References}

[1] I. Suzuki, H.H. Chiu, Multi-droplet combustion of liquid propellants, in: 9th International Symposium on Space Technology and Science, 1971, p. 145.

[2] H.H. Chiu, T.M. Liu, Group combustion of liquid droplets, Combust. Sci. Technol. 17 (1977) 127.

[3] B.T. Zinn, T. Lieuwen, The role of unmixedness in driving combustion instabilities in low $\mathrm{NO}_{x}$ gas turbines, in: 27th Int. Symp. on Comb., 1998, p. 1809.

[4] T.S. Cheng, Y.C. Chao, D.C. Wu, H.W. Hsu, T. Yuan, Effects of partial premixing on pollutant emissions in swirling methane jet flames, Combust. Flame 125 (2001) 865.

[5] M.A. Silverman, D. Dunn-Rankin, Experimental investigation of a rectilinear droplet stream flame, Combust. Sci. Technol. 100 (1994) 57.

[6] C.S. Connon, R. Dimalanta, C. Choi, D. Dunn-Rankin, LIF measurements of fuel vapor in an acetone droplet stream, Combust. Sci. Technol. 129 (1997) 197.

[7] G. Castanet, P. Lavieille, M. Lebouché, F. Lemoine, Measurement of the temperature distribution within monodisperse combusting droplets in linear streams using two-color laser-induced fluorescence, Exper. Fluids 35 (2003) 563.

[8] R. Vehring, G. Schweiger, Optical determination of the temperature of transparent microparticles, Appl. Spectrosc. 46 (1) (1992) 25.

[9] M. Thurber, F. Grisch, B. Kirby, M. Votsmeier, R.K. Hanson, Measurements and modeling of acetone laser-induced fluorescence with implications for temperature-imaging diagnostics, Appl. Opt. 37 (1998) 4963.

[10] A. Lozano, B. Yip, R.K. Hanson, Acetone: a tracer for concentration measurements in gaseous flows by planar laser-induced fluorescence, Exper. Fluids 13 (1992) 369.

[11] M. Orain, X. Mercier, F. Grisch, PLIF imaging of fuel vapour spatial distribution in an acetone droplet stream, comparison with modelling, Combust. Sci. Technol. 177 (2005) 249. 
[12] P. Lavieille, F. Lemoine, G. Lavergne, M. Lebouché, Evaporating and combusting droplet temperature measurements using two color laser induced fluorescence, Exper. Fluids 31 (2001) 45.

[13] G. Koenig, K. Anders, A. Frohn, A new light scattering technique to measure droplet diameter of periodically generated moving droplets, J. Aerosol Sci. 17 (1986) 157.

[14] F. Lemoine, Y. Antoine, M. Wolff, M. Lebouché, Simultaneous temperature and $2 \mathrm{D}$ velocity measurements in a turbulent heated jet using combined laser-induced fluorescence and LDA, Exper. Fluids 26 (1999) 315.

[15] R.K. Hanson, Combustion diagnostics: planar imaging techniques, in: 21st Int. Symp. on Combustion, 1986, p. 1677.

[16] R. Clift, J.R. Grace, M.E. Weber, Bubbles, Drops, and Particles, Academic, New York, 1978.

[17] B. Abramzon, W.A. Sirignano, Droplet vaporization model for spray combustion calculations, Int. J. Heat Mass Transfer 32 (1989) 1605.

[18] G.L. Hubbard, V.E. Denny, A.F. Mills, Droplet evaporation: effects of transients and variable properties, Int. J. Heat Mass Transfer 18 (1975) 1003.
[19] W.A. Sirignano, Fluid Dynamics and Transport of Droplets and Sprays, Cambridge University Press, 1999.

[20] C.H. Chiang, W.A. Sirignano, Interacting, convecting, vaporizing fuel droplets with variable properties, Int. J. Heat Mass Transfer 36 (1993) 875.

[21] G. Castanet, M. Lebouché, F. Lemoine, Heat and mass transfer of combusting monodisperse droplets in linear stream, Int. J. Heat Mass Transfer 48 (2005) 3261.

[22] A. Atthasit, N. Doué, Y. Biscos, G. Lavergne, Influence of droplet concentration on the dynamics and evaporation of a monodisperse stream of droplets in evaporation regime, Advances in Combustion and Atmospheric Pollution, Torus Press, Semenov Memorial, Russia, 2005 , p. 19.

[23] G. Castanet, P. Lavieille, M. Lebouché, F. Lemoine, Experimental and theoretical investigation of the heating of combusting droplets in a linear stream, Combust. Sci. Technol. 177 (2005) 2395.

[24] C.R. Wilke, A viscosity equation for gas mixtures, J. Chem. Phys. 18 (1950) 517. 